\title{
BMJ Open Changes in antipsychotic drug usage and factors affecting the use of typical drugs based on nationwide health insurance data in South Korea
}

\author{
Sang Uk Lee, ${ }^{1,2}$ Vin Ryu, ${ }^{1}$ Minah Soh, ${ }^{1}$ Chul-Eung Kim, ${ }^{1}$ Subin Park, ${ }^{1}$ \\ Sungwon Roh, ${ }^{3}$ In-Hwan Oh, ${ }^{2}$ Hye-Young Lee, ${ }^{1}$ SungKu Choi ${ }^{1}$
}

To cite: Lee SU, Ryu V, Soh M, et al. Changes in antipsychotic drug usage and factors affecting the use of typical drugs based on nationwide health insurance data in South Korea. BMJ Open 2018;8:e020280. doi:10.1136/ bmjopen-2017-020280

- Prepublication history and additional material for this paper are available online. To view these files, please visit the journal online (http://dx.doi. org/10.1136/bmjopen-2017020280).

Received 25 0ctober 2017 Revised 4 May 2018 Accepted 25 May 2018

\section{Check for updates}

${ }^{1}$ Department of Mental Health Research, National Center for Mental Health, Seoul, South

Korea

${ }^{2}$ Department of Preventive Medicine, School of Medicine, Kyung Hee University, Seoul, South Korea

${ }^{3}$ Department of Psychiatry, School of Medicine, Hanyang University, Seoul, South Korea

Correspondence to

Dr SungKu Choi;

clipuni@gmail.com

\section{ABSTRACT}

Objectives To determine changes in antipsychotic drug usage in all South Korean patients with schizophrenia in 2011-2015 and factors affecting their utilisation in 2015.

Design and setting Retrospective cohort study using health insurance claims data on patients with schizophrenia in South Korea in 2011-2015.

Participants All treated patients with schizophrenia in South Korea in 2011-2015. The number of patients with schizophrenia was 171302 in 2011, 175488 in 2012, 177763 in 2013, 180079 in 2014 and 183427 in 2015.

Main outcome measures Changes in antipsychotic drug usage and factors affecting the use of antipsychotics.

Results Among patients with schizophrenia, there were tendencies of decreased use of antipsychotic combination therapy of typical drugs (from $11.5 \%$ to $7.5 \%$ ) but increased use of combination therapy of atypical drugs (from $21.8 \%$ to $29.0 \%$ ). Factors affecting the use of typical drugs were sex, age, geographical region, type of benefits/insurances and type of medical institutions. Use of typical antipsychotics was increased by age $(0 R=1.02$, $95 \% \mathrm{Cl} 1.02$ to 1.02). It was higher in men ( $\mathrm{OR}=1.27$, $95 \% \mathrm{Cl} 1.23$ to 1.30 ) than that in women. It was higher in Medicaid benefiters $(\mathrm{OR}=4.49,95 \% \mathrm{Cl} 4.35$ to 4.64$)$ than that in patients with health insurance. Use of typical drugs was higher among patients treated in general hospitals $(\mathrm{OR}=1.46,95 \% \mathrm{Cl} 1.32$ to 1.64$)$, primary hospitals $(\mathrm{OR}=3.25,95 \% \mathrm{Cl} 2.95$ to 3.59$)$, long-term care hospitals $(\mathrm{OR}=3.00,95 \% \mathrm{Cl} 2.59$ to 3.49$)$ and clinics $(\mathrm{OR}=8.87$, $95 \% \mathrm{Cl} 8.06$ to 9.76 ) compared with that in tertiary care hospitals. Compared with metropolitan region, higher use of typical antipsychotics was seen in Gangwon $(\mathrm{OR}=1.14$, $95 \% \mathrm{Cl} 1.05$ to 1.25$)$, Jeolla ( $\mathrm{OR}=1.32,95 \% \mathrm{Cl} 1.26$ to 1.39) and Gyeongsang $(\mathrm{OR}=1.14,95 \% \mathrm{Cl} 1.10$ to 1.18$)$ provinces.

Conclusions Results of this study confirmed changes of antipsychotic drug usage from typical to atypical antipsychotics in the treatment of schizophrenia and identified factors affecting the use of typical drugs, in contrast with current treatment trend in South Korea. These results may be used in the improvement of a medical system.
Strengths and limitations of this study

This study included all patients treated for schizophrenia in South Korea. Therefore, these results are representative of patients with schizophrenia in South Korea.

- This study confirmed a tendency of antipsychotic drug use in patients with schizophrenia based on their medical records.

- The analysis was conducted by classifying patients into those receiving Medicaid and health insurance to exclude the effect of health insurance system. Therefore, this study showed factors underlying the prescription trend irrespective of socioeconomic status.

- Data of this study did not provide information on the severity of patients' condition. Therefore, we cannot determine changing patterns of prescription based on severity or altered dosage of antipsychotics (defined daily doses)

\section{INTRODUCTION}

Mental disorder is not limited to a specific group, region, country or society. ${ }^{1}$ According to World Economic Forum, mental illness related costs were $\$ 8.5$ billion in 2010 , exceeding costs for cardiovascular disease, cancer and chronic respiratory disease. Costs associated with mental disorders are increasing rapidly. They could double by $2030 .^{2}$

Medical and social costs associated with mental illnesses in South Koreans are very high. ${ }^{34}$ In modern South Korea, $25.4 \%$ of the population had been diagnosed with a mental disorder more than once in their lives. Lifetime prevalence of schizophrenia spectrum disorders is estimated to be $0.5 \%$ among the total population. ${ }^{5}$ Schizophrenia negatively affects a patient's life. ${ }^{6}$ Patients with schizophrenia lack social skills ${ }^{7}$ and display diminished cognitive functions that cause difficulty in adaptation to society and disrupt their normal social support network. ${ }^{8} 9$ Without 
proper treatment, patients have difficulty in living a normal daily life due to decline in function, relapse and worsening symptoms. ${ }^{10}$ To minimise decline in patients' quality of life due to schizophrenia and reduce social cost, aggressive treatment at an early stage is needed.

The most important treatment for schizophrenia is the use of antipsychotics. ${ }^{11}$ Since the advent of chlorpromazine in 1950s, many drugs have been developed. They can be divided into two categories: (1) classical antipsychotic drugs. They work by blocking dopaminergic receptor. However, they can produce involuntary movement disorder by blocking the extrapyramidal system; and (2) atypical antipsychotic drugs. They are effective for schizophrenia. However, they significantly reduce extrapyramidal symptoms by simultaneously blocking dopaminergic and serotonin receptors. The current first-line treatment of schizophrenia is atypical antipsychotics. ${ }^{12}$ Since atypical drugs have less side effects (extrapyramidal symptom and hyperprolactinaemia) compared with typical drugs, drug compliance is high. They can lower relapse due to drug withdrawal despite their relatively high cost. Many studies have reported that atypical antipsychotic drugs are cost-effective considering both direct and indirect costs. ${ }^{13-15}$ The practice guideline for treating patients with schizophrenia in South Korea also recommends atypical antipsychotic drug as a first-line therapy. ${ }^{16}$ Previous research studies on antipsychotic use have only been conducted on patients who are treated in designated hospitals. ${ }^{17-20}$ Thus, antipsychotics use in all patients with schizophrenia in South Korea are currently unknown.

In South Korea, patients are covered by Medicaid and health insurance. Approximately, 96\% of South Korean population are covered by the national health insurance while the rest $(4 \%)$ of the population with an annual assessment of poverty status are covered by a separate programme called Medicaid. Almost all Medicaid patients' treatment is supported by the government financially. ${ }^{21}$ Treatment of patients with schizophrenia differs by the type of health insurance. ${ }^{22}$ However, no study has reported the prescription of antipsychotics by health insurance in South Korea. Thus, the objective of this study was to determine changes of antipsychotic use among patients with schizophrenia in South Korea and factors affecting their usage.

\section{METHODS \\ Data}

This was a retrospective study using health insurance claims data provided by the Health Insurance Review and Assessment (HIRA) from January 2011 to December 2015. In South Korea, $98 \%$ of the population is covered by the national health insurance system. Thus, claims data represent the whole population treated for a specific illness with the exception of those two per cent of citizens living abroad or serving as soldiers. ${ }^{23}$ Presently, a patient with schizophrenia is defined as a person diagnosed with code F20 based on the International Classifcation of Diseases, 10th Revision (ICD-10) disease classification system. Drug specifications records were used to identify drugs prescribed to subjects. Among these drugs, lists of antipsychotic drugs were extracted. Based on the prescription data, drugs were classified into seven categories (typical drug monotherapy, combination therapy of two or more typical drugs, atypical drug monotherapy, combination therapy of two or more atypical drugs, combination therapy of typical and atypical drugs, monotherapy of long-acting injectable antipsychotics and combination therapy of oral antipsychotics and long-acting injectable antipsychotics). Changes in antipsychotic drug usage from 2011 to 2015 were then confirmed. Factors affecting drug use at first visit to medical institutions in 2015 were classified into two categories: typical drug use only (including the use of multiple typical drugs) and atypical drug use only (including the use of multiple atypical drugs). Region data were collected based on the location of medical institutions among 16 cities and provinces (online supplementary figure 1). Medical institutions were categorised into tertiary care hospital, general hospital, primary hospital, long-term care hospital and clinic in accordance with the South Korean classification system. These medical institutions variables are classified based on the number of beds: clinics, $<30$ beds; primary hospitals, 30-99 beds; long-term care hospitals, 30-99 beds, but with focus on nursing; tertiary hospitals, $>99$ beds, and general hospital, >99 beds. The difference between tertiary hospitals and general hospital is quality of medical service. The government evaluates general hospitals and then designates some general hospitals to tertiary hospitals based on results of evaluation. Based on receipt of Medicaid benefit, subjects were divided into Medicaid patients and health insurance patients.

\section{Patient selection}

Among HIRA claim data of 50 million patients who visited medical institutions in South Korea from 2011 to 2015, 96618772 medical records of patients diagnosed as F20 F29 of ICD-10 were extracted. In pooled data, the number of patients with schizophrenia with F20 as a main disease code was 888061. Data for subjects whose age was unidentified were excluded. The number of patients with schizophrenia was 171302 in 2011, 175488 in 2012, 177763 in 2013, 180079 in 2014 and 183427 in 2015 (figure 1). Sociodemographic information on subjects was based on information at the first diagnosis.

\section{Statistical analyses}

In this study, continuous variables are presented as average \pm SD deviation while categorical variables are shown as frequency and percentage. To analyse the difference between groups prescribed with typical and atypical drugs, t-test was performed for continuous variables while $\chi^{2}$ test was performed for categorical variables. Using logistic regression analysis, OR of typical drug use was measured based on demographics. A subgroup analysis was performed by Medicaid benefit to exclude the influence of the Medicaid system in South Korea. OR and 95\% 


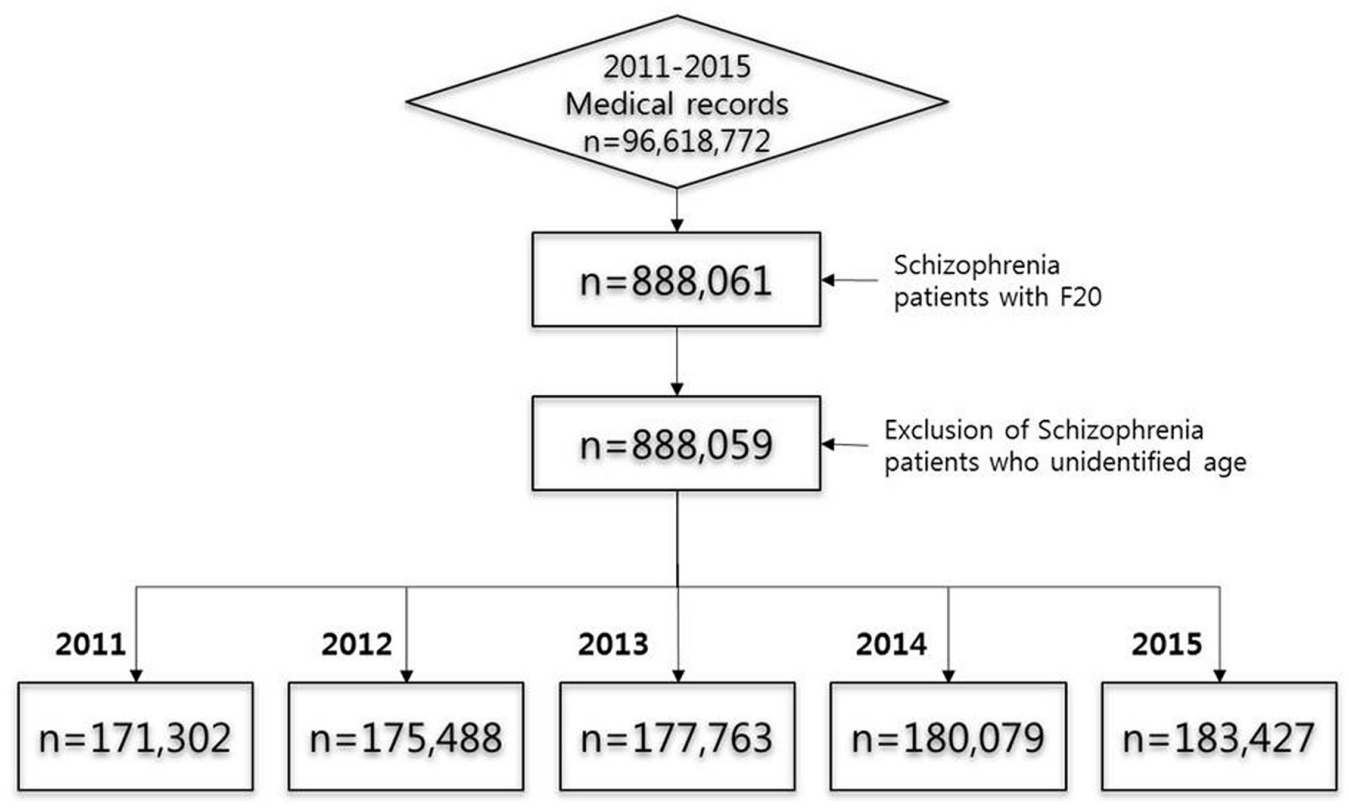

Figure 1 Subject selection flow chart.

CIs were obtained while controlling for sex, age, region, Medicaid benefit and medical institutions. SAS Enterprise Guide V.6.1 was used as all statistical analyses.

\section{Patient involvement}

No patients were involved in developing the hypothesis, the specific aims or the research questions, nor were they involved in developing plans for design or implementation of the study. No patients were involved in the interpretation of study results or write up of the manuscript. There are no plans to disseminate the results of the research to study participants or the relevant patient community.

\section{RESULTS}

Changes in prescription of antipsychotics from 2011 to 2015

The number of patients with schizophrenia was 171302 in 2011. It was increased to 183427 in 2015 . The average age of these patients was 46.2 years in 2011 and 48.5 years in 2015. During this period, the frequency of atypical drug monotherapy was the highest while the frequency of longacting injectable antipsychotics was the lowest. The use of typical drugs was steadily decreased while the number of cases with combination therapy of multiple atypical drugs was continuously increased (table 1).

\section{Sociodemographic characteristics of patients with} schizophrenia taking typical or atypical drugs in $\mathbf{2 0 1 5}$

Patients treated for their schizophrenia in 2015 were classified into two groups; see table 2. More typical drugs were used in men compared with women. They were also used more in older subjects. Medicaid-benefited patients, patients who were treated at primary hospitals, long-term care hospitals, clinics and patients outside the metropolitan region (with the exception of Jeju region) also used more typical drugs than atypical drugs.

Table 1 Number of patients with schizophrenia and antipsychotics prescription pattern in 2011-2015

\begin{tabular}{|c|c|c|c|c|c|}
\hline & 2011 & 2012 & 2013 & 2014 & 2015 \\
\hline Schizophrenia & n (\%)=171302 & n (\%)=175488 & n (\%)=177 763 & n (\%)=180079 & n $(\%)=183427$ \\
\hline Average age (year) & 46.2 & 46.8 & 47.3 & 47.9 & 48.5 \\
\hline First gen. & 195147 (11.04) & 197432 (10.21) & 183554 (9.31) & $165606(8.30)$ & $151860(7.53)$ \\
\hline $1+1$ combi & 203485 (11.51) & $193162(9.99)$ & $180141(9.14)$ & $165250(8.28)$ & 150459 (7.46) \\
\hline Second gen. & 595689 (33.70) & 661154 (34.20) & 663118 (33.63) & $660788(33.13)$ & 656942 (32.58) \\
\hline $2+2$ combi & 385061 (21.79) & 450116 (23.28) & 492309 (24.97) & $541465(27.14)$ & 584469 (28.99) \\
\hline LAl+drug & 36279 (2.05) & $40438(2.09)$ & $42236(2.14)$ & $44989(2.26)$ & $47968(2.38)$ \\
\hline LAl & $3103(0.18)$ & $9839(0.51)$ & $13092(0.66)$ & $15175(0.76)$ & $16153(0.80)$ \\
\hline
\end{tabular}

Combi, Combination therapy of two or more antipsychotics drugs; LAI, Long-acting injection. 
ORs of typical drug use by sociodemographic characteristics

When we analysed the use of typical antipsychotics taking sociodemographic characteristics into account, statistically significant differences were found for sex, age, geographical region, type of benefit/insurance and type of medical institutions (table 3). Men were prescribed typical antipsychotics more often than women $(\mathrm{OR}=1.27$, 95\% CI 1.23 to 1.30). Regarding age, OR of typical drug use was 1.02 (95\% CI 1.02 to 1.02) which was high as age was increased by year. In terms of regions, three provinces (Gangwon, Jeolla and Geyongsang) displayed higher and one place (Jeju) showed lower usage of typical antipsychotics when compared with metropolitan region (table 3). OR of typical drug use in Medicaid patients was 4.49 (95\% CI 4.35 to 4.64 ), which was higher compared with that of health insurance patients. In terms of medical institutions compared with tertiary hospitals, ORs of typical drug use in general hospitals, primary hospitals, long-term care hospitals and clinics were 1.47 (95\% CI
1.32 to 1.64$), 3.26$ (95\% CI 2.95 to 3.59$), 3.00$ (95\% CI 2.59 to 3.49$)$ and 8.87 (95\% CI 8.06 to 9.76$)$, respectively.

ORs of typical drug use by sociodemographic factor of patients on health insurance and Medicaid

After adjusting for sociodemographic factors, ORs of typical drug use by sex, age, region and medical institutions in patients on health insurance and Medicaid were determined. ORs of typical drug use in men compared with women were 1.23 (95\% CI 1.18 to 1.27 ) and 1.34 (95\% CI 1.28 to 1.41) in the health insurance group and the Medicaid group, respectively. Thus, typical drug use in both groups was significantly higher in men than that in women (figure 2).

In terms of OR by age in health insurance patients, compared with patients $\leq 19$ years of age, ORs of typical drug use in patients $30-39,40-49,50-59,60-69,70-79$ and $\geq 80$ years of age were 2.06 (95\% CI 1.55 to 2.72), 4.66 (95\% CI 3.54 to 6.13 ), 7.83 (95\% CI 5.95 to 10.30), 7.41

Table 2 Sociodemographic factors of patients with schizophrenia divided with regard to the type of antipsychotic used

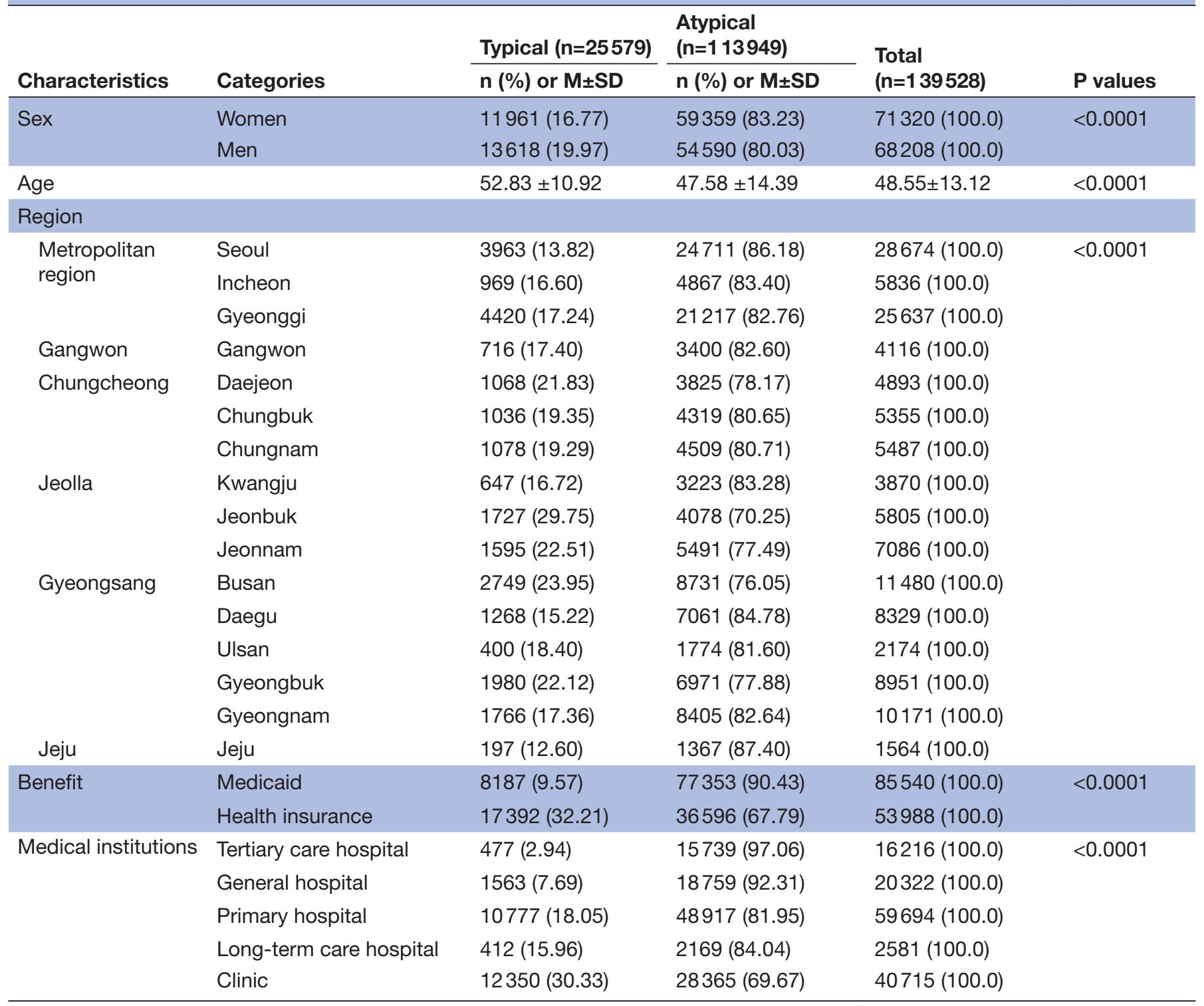


Table 3 OR of typical drug use by sociodemographic characteristics

\begin{tabular}{|c|c|c|c|c|}
\hline Characteristics & Categories & Typical & Atypical & OR (95\% Cl) \\
\hline \multirow[t]{2}{*}{$\operatorname{Sex}^{*}$} & Women & 11961 & 59359 & 1.00 (reference) \\
\hline & Men & 13618 & 54590 & 1.27 (1.228 to 1.303$)$ \\
\hline \multirow[t]{5}{*}{ Region $\ddagger$} & Metropolitan region & 9352 & 50795 & 1.00 (reference) \\
\hline & Gangwon & 716 & 3400 & $1.14(1.045$ to 1.254$)$ \\
\hline & Jeolla & 3969 & 12792 & 1.32 (1.264 to 1.386$)$ \\
\hline & Gyeongsang & 8163 & 32942 & 1.14 (1.097 to 1.179$)$ \\
\hline & Jeju & 197 & 1367 & $0.62(0.530$ to 0.733$)$ \\
\hline \multirow[t]{2}{*}{ Benefit§ } & Health insurance & 8187 & 77353 & 1.00 (reference) \\
\hline & Medicaid & 17392 & 36596 & 4.49 (4.349 to 4.641$)$ \\
\hline \multirow{3}{*}{ Medical institutions } & Primary hospital & 10777 & 48917 & 3.26 (2.951 to 3.590$)$ \\
\hline & Long-term care hospital & 412 & 2169 & 3.00 (2.587 to 3.485$)$ \\
\hline & Clinic & 12350 & 28365 & 8.87 (8.060 to 9.762$)$ \\
\hline
\end{tabular}

${ }^{*}$ Adjusted for age, region, medical institutions and Medicaid benefit. †Adjusted for sex, region, medical institutions and Medicaid benefit. $\ddagger$ Adjusted for sex, age, medical institutions and Medicaid benefit. $\S$ Adjusted for sex, age, region and medical institutions.

ๆAdjusted for sex, age, region and Medicaid benefit.

(95\% CI 5.61 to 9.78$), 5.34$ (95\% CI 4.00 to 7.12 ) and 3.36 (95\% CI 2.44 to 4.64 ), respectively. In Medicaid patients compared with patients $\leq 19$ years of age, ORs of typical drug use in patients 20-29, 30-39, 40-49, 50-59, 60-69, $70-79$, and $\geq 80$ years of age were 1.62 (95\% CI 1.15 to 2.29), 2.13 (95\% CI 1.54 to 2.95 ), 3.03 (95\% CI 2.20 to 4.17 ), 4.00 (95\% CI 2.95 to 5.30$), 3.88$ (95\% CI 2.61 to 4.78), 2.60 (95\% CI 2.00 to 3.12 ) and 1.83 (95\% CI 1.44 to 2.64 ), respectively (figure 3 ).

In terms of OR of typical drug use in health insurance patients by region, compared with metropolitan region,

\section{Health insurance patients}

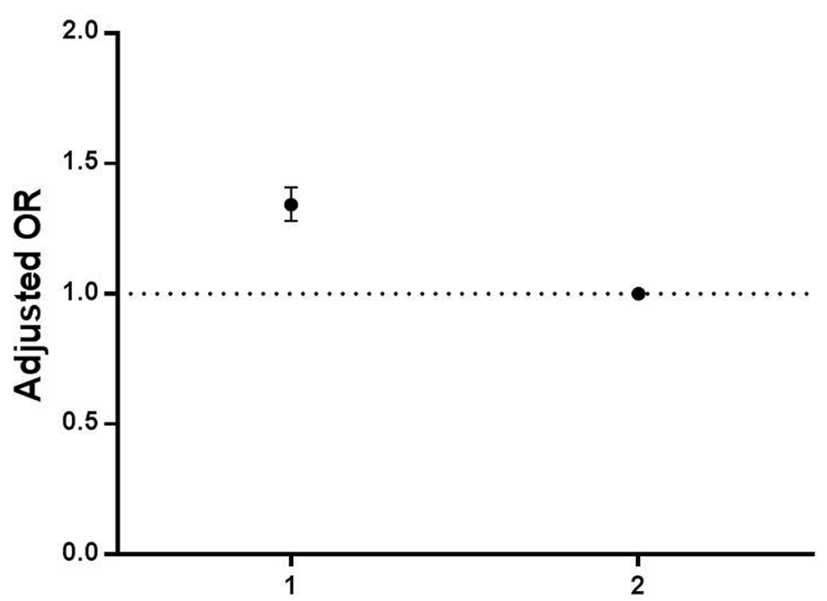

ORs in Gangwon, Jeolla and Gyeongsang provinces were 1.19 (95\% CI 1.03 to 1.39 ), 1.23 (95\% CI 1.14 to 1.33 ) and 1.20 (95\% CI 1.14 to 1.27$)$, respectively. The OR of 0.57 (95\% CI 0.44 to 0.73 ) in Jeju was quite low. ORs of typical drug use in Medicaid patients in Chungcheong, Jeolla and Gyeongsang provinces were 1.09 (95\% CI 1.02 to 1.16$), 1.35$ (95\% CI1.26 to 1.43 ) and 1.10 (95\% CI 1.05 to 1.15 ), respectively, compared with that in metropolitan region. It was 0.67 (95\% CI 0.54 to 0.83 ) in Jeju (figure 4).

In terms of OR of typical drug use in health insurance patients by medical institutions, compared with

\section{Medicaid patients}

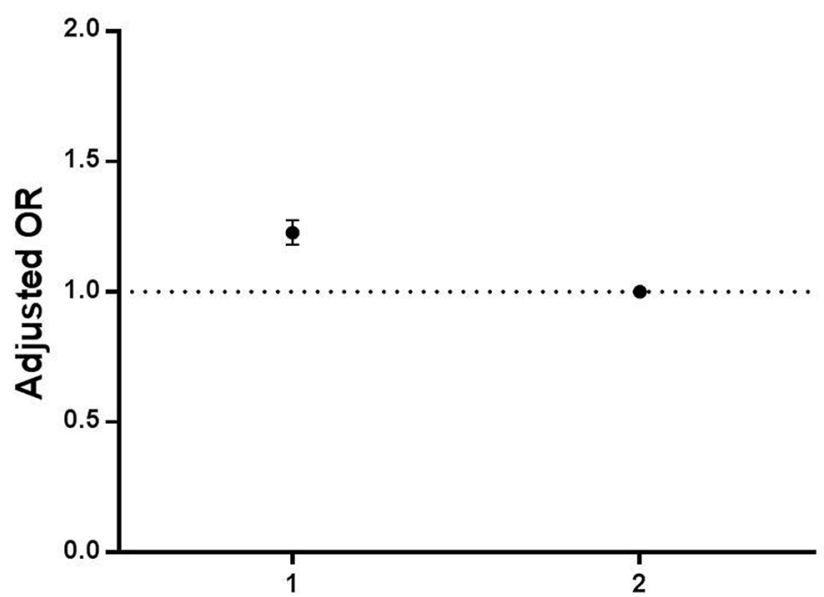

Figure 2 Typical drug use by sex in patients divided by the type of health insurance coverage. 1: Men, 2: Women. Adjusted for age, region and medical institutions. 
Health insurance patients

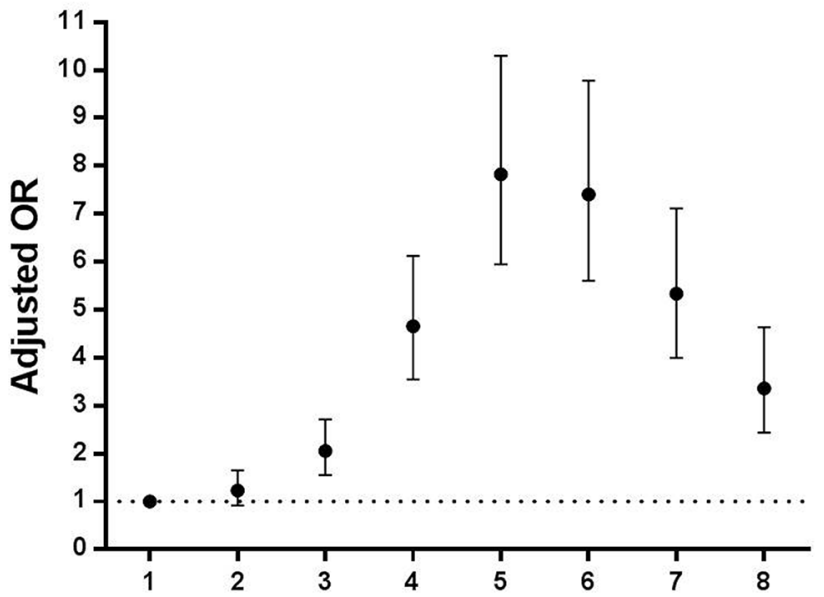

Medicaid patients

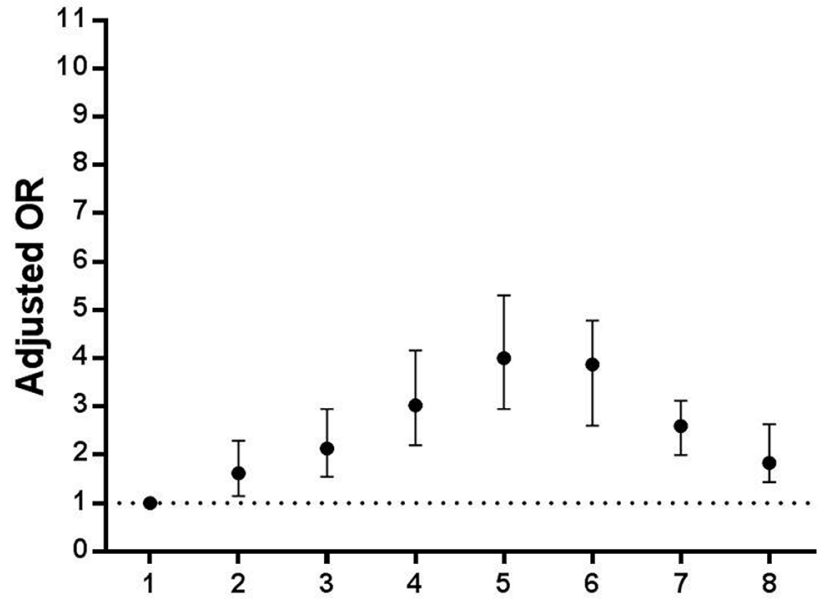

Figure 3 Typical drug use by age in patients divided by the type of health insurance coverage. 1: $\leq 19$ years, 2: 20-29 years, 3: 30-39 years, 4: 40-49 years, 5: 50-59 years, 6: 60-69 years, 7: 70-79 years and 8: $\geq 80$ years. Adjusted for sex, region and medical institutions.

tertiary care hospitals, ORs in general hospitals, primary hospitals, long-term care hospitals and clinics were 1.35 (95\% CI 1.18 to 1.55 ), 2.95 (95\% CI 2.62 to 3.31 ), 2.00 (95\% CI 1.54 to 2.59$)$ and 8.66 (95\% CI 7.76 to 9.67$)$, respectively. In Medicaid patients, compared with tertiary care hospital, ORs in general hospitals, primary hospitals, long-term care hospitals and clinics were 1.77 (95\% CI 1.44 to 2.17 ), 3.94 (95\% CI 3.24 to 4.80 ), 4.14 (95\% CI 3.27 to 5.24 ) and 10.22 (95\% CI 8.41 to 12.43 ), respectively (figure 5).

\section{DISCUSSION}

This study was conducted on patients with schizophrenia in South Korea from 2011 to 2015 using HIRA database to investigate changes in antipsychotic use in South Korea and statistically analyse the impact of demographic factors on typical antipsychotic use.

\section{Health insurance patients}

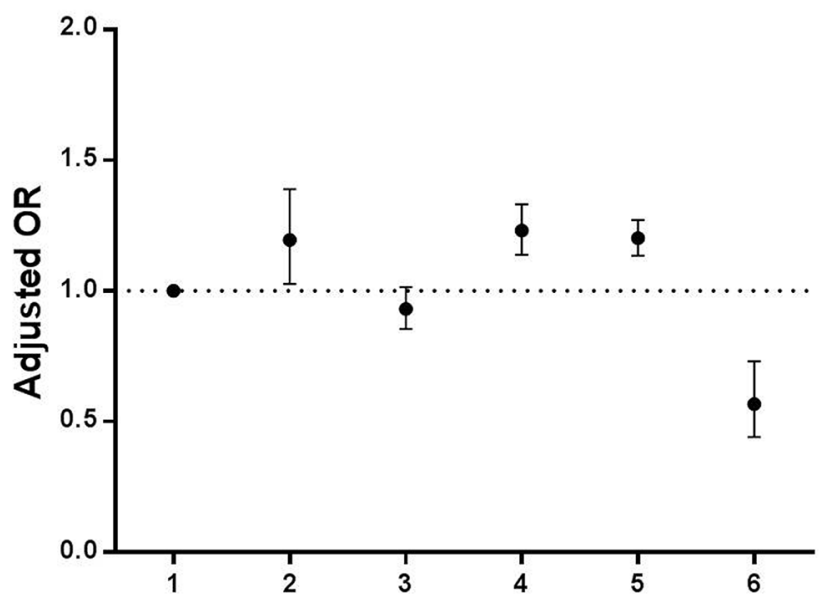

The number of patients diagnosed with schizophrenia in South Korea was increased from 171302 in 2011 to 183427 in 2015 . Such increase may have been due to decline in mortality of patients with schizophrenia. It has been reported that the death rate of patients with schizophrenia is increased continuously compared with the general population, peaking in 1995 followed by subsequent decline. ${ }^{24}$ Thus, the increase shown in this study likely reflects recent decreased death rates in this patient group. The average age of patients with schizophrenia included in this study was increased from 46.2 years in 2011 to 48.5 years in 2015 , coinciding with the decline in death rate.

The use of typical drugs in patients with schizophrenia was decreased from 2011 to 2015. Meanwhile, the use of combination therapy of two or more atypical drugs has increased. Increase in atypical antipsychotic use has been

\section{Medicaid patients}

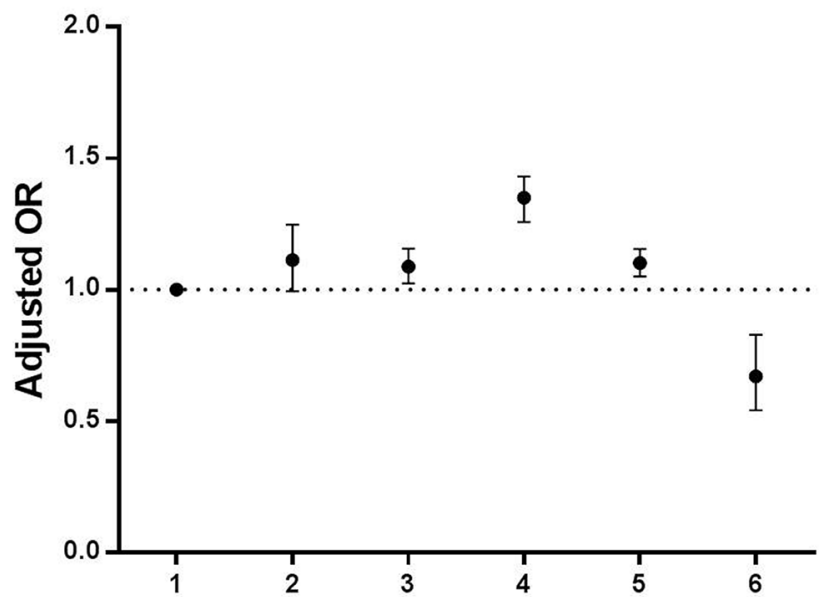

Figure 4 Typical drug use by region in patients divided by the type of health insurance coverage. 1: Metropolitan region, 2: Gangwon province, 3: Chungcheong province, 4: Jeolla province, 5: Gyeongsang province and 6: Jeju island. Adjusted for sex, age and medical institutions. 


\section{Health insurance patients}

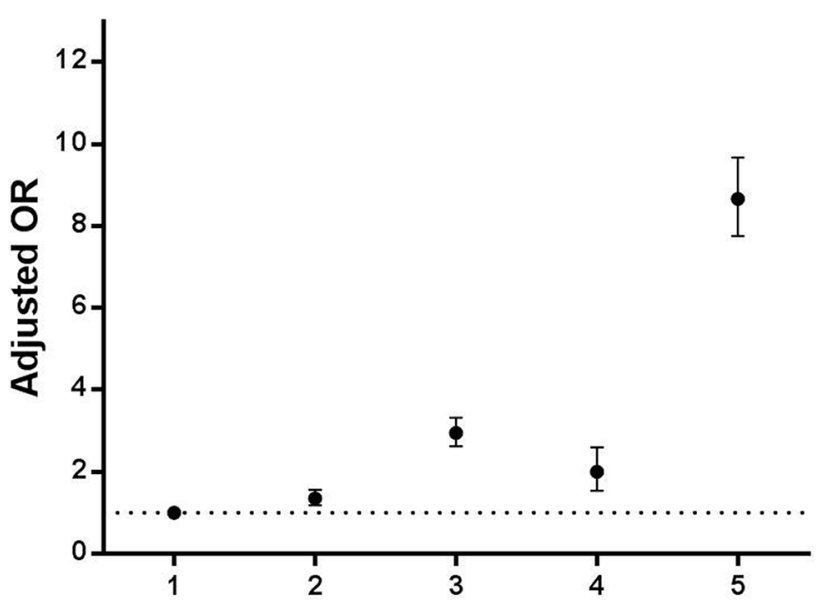

Medicaid patients

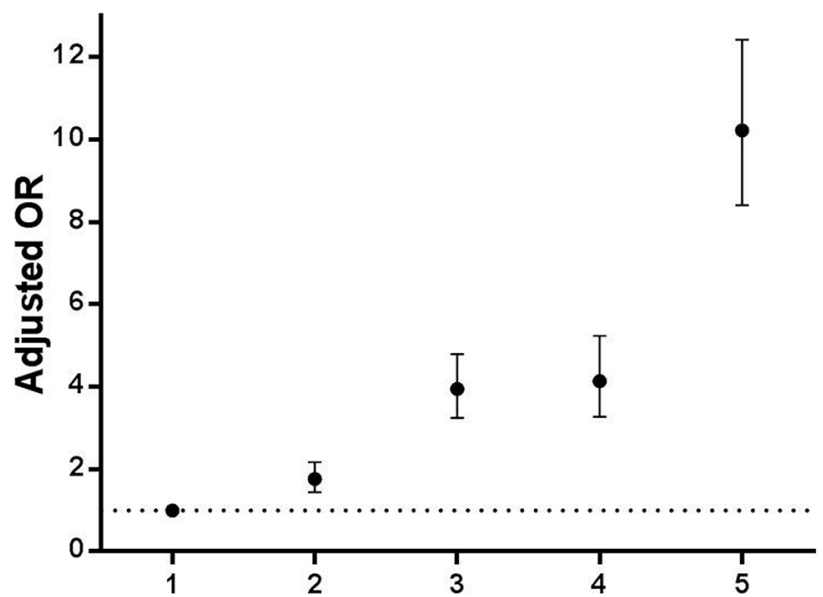

Figure 5 Typical drug use by medical institutions in patients divided by the type of health insurance coverage. 1: Tertiary care hospital, 2: General hospital, 3: Primary hospital, 4: Long-term care hospital, and 5: Clinic. Adjusted for sex, age and region.

reported in many countries. ${ }^{25}{ }^{26} \mathrm{~A}$ unique feature in the pattern of atypical drug use was the increased frequency of combination therapy containing two or more atypical drugs, although there was no big change in the use of atypical antipsychotic monotherapy. Pickar et al have reported an increase in multidrug therapy combining two or more antipsychotics for patients with schizophrenia. ${ }^{27}$ This pattern might have resulted in the improvement of symptoms and efficacy. ${ }^{28}{ }^{29}$ Other studies conducted in South Korea have also shown that combination therapies of two or more atypical drugs have the highest frequency among prescription patterns involving antipsychotics. ${ }^{30}$

The significantly higher use of typical antipsychotics in men than in women might be attributed to side effects of antipsychotics. Antipsychotics are known to increase prolactin level which may cause amenorrhoea that is especially higher in patients treated with typical drugs. ${ }^{31}$ Therefore, women who are at risk of amenorrhoea clinically might be prescribed typical antipsychotics more often than men.

In the present study, the use of typical antipsychotics was increased with age, peaking in the age group of 50-60 years with subsequent decline thereafter. In South Korea, typical drugs were introduced in 1990s. However, the use of atypical drugs was recommended as a first-line treatment for schizophrenia in $2006 .{ }^{16}$ In other words, patients with schizophrenia usually received typical drugs before 2006, indicating that patients older than 30-40 years might have been exposed to them. A change in drug therapy is indicated when patients show partial or no response to mainstream treatment. ${ }^{32}$ Thus, if patients who were previously treated with typical drugs have no reason to switch medications, they are likely to continue treatment with typical drugs. However, the number of typical drug prescriptions was decreased at $60-70$ years of age. This suggests that the disease might have caused a functional decline, relapse and worsening of symptoms with increasing age. ${ }^{33}$ Increased age and worsening symptoms may prompt changes in atypical drug usage or use of multiple drugs.

Currently, the pattern of OR of typical drug use in patients with schizophrenia varies by geographical region. Compared with metropolitan regions, increased use of typical drugs was seen in Gangwon province $(\mathrm{OR}=1.14)$, Jeolla province $(\mathrm{OR}=1.32)$ and Gyeongsang province $(\mathrm{OR}=1.14)$ while a decreased use was found in Jeju island $(\mathrm{OR}=0.623)$. Clinical experience and preferences of medical doctors can also affect drug prescription pattern. ${ }^{34}$ This might be a possible explanation for such geographical differences observed in this study.

The use of typical antipsychotics was significantly higher in Medicaid patients compared with that in patients receiving health insurance $(\mathrm{OR}=4.49)$. Typical drug use was also significantly higher in general hospitals $(\mathrm{OR}=1.46)$, primary hospitals $(\mathrm{OR}=3.25)$, long-term care hospitals $(\mathrm{OR}=3.00)$ and clinics $(\mathrm{OR}=8.87)$ compared with tertiary care hospitals. Especially, the OR of typical drug use in clinics was ninefold higher than that in a tertiary care hospital setting. This pattern might be attributed to the current South Korean medical and health insurance systems. In the South Korean medical system, the ratio of private medical institutions is about $94 \% .{ }^{35}$ In the health insurance system, the medical cost per day is fixed for Medicaid patients with schizophrenia regardless of the therapy provided. ${ }^{36}$ When the price is converted to cost per day, costs for inpatient's and outpatient's treatment are 2770 won (about US\$2.4) and 50000 won (about US\$44.1), respectively. ${ }^{37}$ In this system, medical institutions are required to use low-cost drugs in order to realise profits. Use of high-cost drugs or expensive labour may shrink profit margins. For the average treatment cost of Medicaid patients in South Korea, only about $65 \%$ of the cost is covered by health insurance. This indicates that low-cost therapy is provided to Medicaid patients. ${ }^{22}$ As medical expenses are directly related to profit, such tendency is especially prominent in clinics. However, it 
was hard to conclude that our result was only affected by the fixed-cost payment system as suggested in previous studies. ${ }^{35}$ When we analysed Medicaid patients and health insurance patients separately, a similar prescription pattern occurred in both groups following a geographic pattern. This suggests that the experience and preferences of clinicians at different medical institutions might have affected the prescription pattern as well. ${ }^{34} 38$

This study has several limitations. First, HIRA data did not provide the actual status of patients with schizophrenia. For example, HIRA data do not include the severity of the disease. However, drug prescription patterns in schizophrenia outpatients differ according to the severity of the disease. ${ }^{39}$ Therefore, clinical factors influencing antipsychotic prescription patterns in schizophrenia were not comprehensively analysed. Second, in this study, factors affecting the use of typical drugs were not analysed, including prescription period, prescription changes or dosage of antipsychotics. Although we analysed factors affecting the use of antipsychotics in patients with schizophrenia visiting medical institutions in 2015 for the first time, further research should consider these limitations.

Despite these limitations, our study has a number of strengths. Using HIRA data, this study confirmed changes in antipsychotic usage in all patients in South Korea with schizophrenia and factors affecting their usage. Another strength is that a number of sociodemographic factors such as sex, age, geographical region, type of benefit/ insurance and type of medical institutions known to influence prescription pattern are taken into account. These findings can be used as evidence to improve the medical system and strengthen continuing education programmes for the treatment of patients with schizophrenia.

\section{CONCLUSIONS}

In this study, HIRA data were analysed to identify changes in antipsychotic usage and factors affecting the drug use in patients with schizophrenia in South Korea. In terms of drug prescription, a decrease in typical drug use and increase in atypical drug use were observed. Factors that significantly affected the use of typical antipsychotics were sex, age, geographical region, type of benefit/insurance and type of medical institutions. Results of this study can be used as evidence to improve the medical system, strengthen continuing education and set future directions for the treatment of patients with schizophrenia.

Contributors SUL and SC developed the idea for the study and design of the search strategy. SUL, VR and I-HO performed the statistical analyses. SUL, C-EK, SP and SR contributed to the first and final draft of the manuscript. SUL, MS, H-YL and SC contributed to the interpretation of the study results. All authors contributed to data interpretation, reviewed successive drafts and approved the final version of the manuscript.

Funding This study was supported by the South Korean Mental Health Technology R\&D Project funded by the Ministry of Health \& Welfare, Republic of Korea. This study was supported by a grant (HM14C-2166) of the Korean Mental Health Technology R\&D Project funded by the Ministry of Health \& Welfare, Republic of Korea.
Disclaimer The sponsor of the study had no role in study design, data collection, data analysis, data interpretation or writing of the report.

Competing interests None declared.

Patient consent Not required.

Ethics approval This study was approved by Institutional Review Board of the National Center for Mental Health of Korea (approval number: 116271-2016-28). Because this study analysed publicly available, anonymised and deidentified data, informed consents were waived.

Provenance and peer review Not commissioned; externally peer reviewed.

Data sharing statement According to Health Insurance Review \& Assessment data protection regulation, administrative data are made available for specific research projects. Thus, we cannot share the data we used for this study with other researchers. However, we can answer any questions about the data used in this study.

Open access This is an open access article distributed in accordance with the Creative Commons Attribution Non Commercial (CC BY-NC 4.0) license, which permits others to distribute, remix, adapt, build upon this work non-commercially, and license their derivative works on different terms, provided the original work is properly cited and the use is non-commercial. See: http://creativecommons.org/ licenses/by-nc/4.0/

(c) Article author(s) (or their employer(s) unless otherwise stated in the text of the article) 2018. All rights reserved. No commercial use is permitted unless otherwise expressly granted.

\section{REFERENCES}

1. World Health Organization. Prevention of Mental Disorders: Effective Interventions and Policy Options. Geneva: World Health Organization, 2004:15-16.

2. Bloom D, Cafiero E, Jané-Llopis E, et al. The global economic burden of noncommunicable diseases. Geneva: World Economic Forum, 2012.

3. Chang SM, Cho SJ, Jeon HJ, et al. Economic burden of schizophrenia in South Korea. J Korean Med Sci 2008;23:167-75.

4. Kwon OY. Mental illness sector $R$ \& $D$ trends and implications. Osong: Industry KHID, 2013:134-9.

5. Hong JP, Lee DW, Ham BJ, et al; The epidemiological survey of mental disorders in Korea. Sejong: Ministry of Health \& Welfare, 2016:15-18.

6. McGrath J, Saha S, Chant D, et al. Schizophrenia: a concise overview of incidence, prevalence, and mortality. Epidemiol Rev 2008;30:67-76.

7. Bellack AS, Morrison RL, Wixted JT, et al. An analysis of social competence in schizophrenia. Br J Psychiatry 1990;156:809-18.

8. Halford WK, Hayes RL. Social skills in schizophrenia: assessing the relationship between social skills, psychopathology and community functioning. Soc Psychiatry Psychiatr Epidemiol 1995;30:14-19.

9. Macdonald EM, Jackson HJ, Hayes RL, et al. Social skill as determinant of social networks and perceived social support in schizophrenia. Schizophr Res 1998;29:275-86.

10. Marengo JT, Harrow M, Westermeyer JF. Early longitudinal course of acute-chronic and paranoid-undifferentiated schizophrenia subtypes and schizophreniform disorder. J Abnorm Psychol 1991;100:600-3.

11. Cassidy CM, Norman R, Manchanda R, et al. Testing definitions of symptom remission in first-episode psychosis for prediction of functional outcome at 2 years. Schizophr Bull 2010;36:1001-8.

12. Lieberman JA, Stroup TS, McEvoy JP, et al. Effectiveness of antipsychotic drugs in patients with chronic schizophrenia. $N$ Engl J Med 2005;353:1209-23.

13. Datta SS, Kumar A, Wright SD, et al. Evidence base for using atypical antipsychotics for psychosis in adolescents. Schizophr Bull 2014;40:252-4.

14. Vishal S, Beg MA, Dutta SB, et al. Comparative evaluation of costeffectiveness between typical antipsychotic haloperidol and atypical antipsychotics olanzapine, risperidone and aripiprazole in the treatment of stable schizophrenia. International Journal of Basic \& Clinical Pharmacology 2017;6:1965-8.

15. Valenstein M, Ganoczy D, McCarthy JF, et al. Antipsychotic adherence over time among patients receiving treatment for schizophrenia: a retrospective review. J Clin Psychiatry 2006;67:1542-50.

16. Bae SO, Ahn YM, Kwon JS, et al. The Feasibility Test of Korean Medication Algorithm for the Treatment with Schizophrenic Patients 
(I): Analysis Focusing on the Effectiveness of Treatment. Korean $J$ Psychopharmacol 2006;17:24-34.

17. Choi HJ, Jung SH, Kang MH, et al. Antipsychotics prescribing patterns of patients with schizophrenia admitted to Korean general hospital psychiatric unit: 2001 to 2008. Clin Psychopharmacol Neurosci 2011;9:17-22.

18. Choi J, Kang SH, Lee Jl, et al. Clinical Correlates and Description Profiles of Antipsychotic Polypharmacy for Patients with Schizophrenia. J Korean Neuropsychiatr Assoc 2014;53:370-8.

19. Kim W, Woo YS, Bahk WM, et al. Changes in the antipsychotic drugs usage in the psychiatric inpatients at a university hospital between 1997 and 2003. Korean J Psychopharmacol 2005;16:475-9.

20. Kwon JS, Kim ET, Th $\mathrm{H}$, et al. Drug prescribing patterns of outpatients with schizophrenia in a university hospital. $J$ Korean Neuropsychiatr Assoc 2003;42:683-90.

21. Kim JH, Lee KS, Yoo KB, et al. The differences in health care utilization between Medical Aid and health insurance: a longitudinal study using propensity score matching. PLoS One 2015;10:e0119939.

22. Park JI. Present and future of mental health policy in Korea. Monthly Welfare Trends 2014:188:5-9.

23. Kim L, Sakong J, Kim Y, et al. Developing the Inpatient Sample for the National Health Insurance Claims Data. Health Policy and Management 2013;23:152-61.

24. Bushe CJ, Taylor M, Haukka J. Mortality in schizophrenia: a measurable clinical endpoint. J Psychopharmacol 2010;24:17-25.

25. Gilmer TP, Dolder CR, Folsom DP, et al. Antipsychotic polypharmacy trends among Medicaid beneficiaries with schizophrenia in San Diego County, 1999-2004. Psychiatr Serv 2007;58:1007-10.

26. Li Q, Xiang YT, Su YA, Ya S, et al. Antipsychotic polypharmacy in schizophrenia patients in China and its association with treatment satisfaction and quality of life: findings of the third national survey on use of psychotropic medications in China. Aust N Z J Psychiatry 2015:49:129-36.

27. Pickar D, Vinik J, Bartko JJ. Pharmacotherapy of schizophrenic patients: preponderance of off-label drug use. PLoS One 2008;3:e3150.
28. Correll CU, Rummel-Kluge C, Corves C, et al. Antipsychotic combinations vs monotherapy in schizophrenia: a meta-analysis of randomized controlled trials. Schizophr Bull 2009;35:443-57.

29. Zink M. Augmentation of olanzapine in treatment-resistant schizophrenia. J Psychiatry Neurosci 2005;30:409.

30. Chang JG, Roh D, Kim CH. Recent trends of antipsychotics polypharmacy in schizophrenia. Korean J Psychopharmacol 2013;24:137-46.

31. Madhusoodanan S, Parida S, Jimenez C. Hyperprolactinemia associated with psychotropics-a review. Hum Psychopharmacol 2010;25:281-97.

32. Ahn YM, Kwon JS, Bahk WM, et al. The feasibility test of Korean medication algorithm for the treatment with schizophrenic patients (II): the problem for applying algorithm to the real clinical situation and opinion of revision. Korean J Psychopharmacol 2006;17:35-49.

33. Müller N. Mechanisms of relapse Prevention in Schizophrenia. Pharmacopsychiatry 2004;37:141-7.

34. Kishimoto $\mathrm{T}$, Watanabe $\mathrm{K}$, Uchida $\mathrm{H}$, et al. Antipsychotic polypharmacy: a Japanese survey of prescribers' attitudes and rationales. Psychiatry Res 2013;209:406-11.

35. Lee YM. Health policy for the people. J Korean Med Assoc 2017;60:286-8.

36. Kim AM. Why do psychiatric patients in Korea stay longer in hospital? IJMHS 2017;11:2.

37. Roh OH, Lee CH, Park A, et al. A Study on Characteristics of Medical Expenses and the Hospitalization Period of Hospitalized Patients Using Diem Payment System at Convalescent Hospitals. J Korea Acad Industr Coop Soc 2016;17:407-14.

38. Jeong SH, Ahn YM, Kim YS. The Variations in the Treatment Pattern of Schizophrenic Patients with Risperidone and Olanzapine. J Korean Neuropsychiatr Assoc 2003;42:79-88.

39. Weinbrenner S, Assion HJ, Stargardt T, et al. Drug prescription patterns in schizophrenia outpatients: analysis of data from a German health insurance fund. Pharmacopsychiatry 2009;42:66-71. 